This item was submitted to Loughborough's Research Repository by the author.

Items in Figshare are protected by copyright, with all rights reserved, unless otherwise indicated.

\title{
Dynamical density functional theory: phase separation in a cavity and the influence of symmetry
}

PLEASE CITE THE PUBLISHED VERSION

http://dx.doi.org/10.1088/0953-8984/17/45/009

PUBLISHER

(C) IOP Publishing

VERSION

SMUR (Submitted Manuscript Under Review)

\section{PUBLISHER STATEMENT}

This work is made available according to the conditions of the Creative Commons Attribution-NonCommercialNoDerivatives 4.0 International (CC BY-NC-ND 4.0) licence. Full details of this licence are available at: https://creativecommons.org/licenses/by-nc-nd/4.0/

\section{LICENCE}

CC BY-NC-ND 4.0

\section{REPOSITORY RECORD}

Archer, Andrew J.. 2019. "Dynamical Density Functional Theory: Phase Separation in a Cavity and the Influence of Symmetry". figshare. https://hdl.handle.net/2134/15979. 


\title{
Dynamical density functional theory: phase separation in a cavity and the influence of symmetry
}

\author{
A J Archer \\ H H Wills Physics Laboratory, University of Bristol, Bristol BS8 1TL, UK
}

\begin{abstract}
Consider a fluid composed of two species of particles, where the interparticle pair potentials $u_{11}=u_{22} \neq u_{12}$. On confining an equal number of particles from each species in a cavity, one finds that the average one body density profiles of each species are constrained to be exactly the same due to the symmetry, when both external cavity potentials are the same. For a binary fluid of Brownian particles interacting via repulsive Gaussian pair potentials that exhibits phase separation, we study the dynamics of the fluid one body density profiles on breaking the symmetry of the external potentials, using the dynamical density functional theory of Marconi and Tarazona [J. Chem. Phys., 110, 8032 (1999)]. On breaking the symmetry we see that the fluid one body density profiles can then show the phase separation that is present.
\end{abstract}

In equilibrium density functional theory (DFT) [1, the key quantity is the Helmholtz free energy functional. Given this functional, one can calculate the (ensemble) average one body density profile(s) for a fluid of particles subject to any given external potential. The form of the Helmholtz free energy functional depends, of course, upon the particular interaction potentials between the particles in the fluid. However, this functional is in general unknown, except in a few cases for 1-dimensional fluids [1. None the less, in the last 30 years, a number of rather accurate approximate Helmholtz free energy functionals have been developed for a wide class of fluids - see Ref. [1] and references therein. When attempting to construct a theory for the dynamics of the (ensemble) average density profile of a fluid confined in a time dependent external potential, it is very appealing, given this large body of work, to base a theory for the dynamics upon the equilibrium Helmholtz free energy functional. In other words, if one is wanting to study the dynamics of a fluid, is it possible to just apply the relevant equilibrium Helmholtz free energy functional in the dynamical theory?

Recently, Marconi and Tarazona derived a dynamical density functional theory (DDFT) 2, 3] for fluids of Brownian particles. Their theory is based upon the equilibrium fluid Helmholtz free energy functional in a rather simple way and therefore has attracted much interest. The theory is not exact; it involves approximating the two body correlations in the non-equilibrium fluid by those of an equilibrium fluid with the same one body density profile [2, 3, 4]. However, for all the cases where comparison with Brownian dynamics (BD) simulations has been made, the theory has proved to be remarkably accurate [2, 5, 6, 7, 8]. It appears that as long as one has an accurate 
approximation for the equilibrium Helmholtz free energy functional, then the DDFT gives a good account of the dynamics for Brownian fluids.

The present paper builds upon an earlier study [7, in which we considered two different asymmetric binary mixtures of particles interacting via repulsive Gaussian pair potentials [9]: $u_{i j}(r)=\epsilon_{i j} \exp \left(-r^{2} / R_{i j}^{2}\right)$, where $i, j=1,2$ label the two different species of particles, $\epsilon_{i j}>0$ is a parameter that determines the strength and $R_{i j}$ denotes the range of the interaction potential. For different choices of these parameters one can obtain either a mixture exhibiting bulk fluid-fluid (macro)phase separation [10, 11, 7], or alternatively, a mixture exhibiting microphase separation [12, 7]. We found that the DDFT used in conjunction with an extremely simple approximate (RPA) Helmholtz free energy functional was able to account accurately for the dynamics of phase separation induced by reducing the radius of the confining spherical cavity potential [7]. In the present paper we consider similar situations but for symmetric binary Gaussian core model (GCM) fluids, i.e. where $R_{11}=R_{22}$ and $\epsilon_{11}=\epsilon_{22}$, but where $R_{12} \neq R_{11}$ or $\epsilon_{12} \neq \epsilon_{11}$. The presence of this symmetry can have a dramatic effect on the confined fluid one body density profiles in cases where there are equal numbers of particles for each species. As long as the confining cavity potentials are the same for both species of particles, then the ensemble (or time) average one body density profiles of the two species must be exactly the same. Symmetry dictates this must always be the case, even when the fluid exhibits phase separation. Here we show that when one breaks the symmetry of the confining cavity potential, such that the cavity walls favour one of the species, then the fluid one body density profiles can change significantly and show the influence of the phase separation - see for example the results in Figs. 1 4 ,

We now describe further the model fluid and the DDFT: The GCM fluid pair potentials have no hard core and the centres of the particles can overlap completely. Such potentials arise when considers the effective potential between the centres of mass of polymers in a good solvent, see Ref. [9] and references therein. In this case $\epsilon_{i j} \sim 2 k_{B} T$ and $R_{i j} \sim R_{g}$, the polymer radius of gyration. We consider two symmetric binary mixtures of GCM particles. In both cases $\epsilon_{11}=\epsilon_{22}=\epsilon_{12}=2 k_{B} T$ and $R_{11}=R_{22}$. The first mixture, which we denote "fluid A", is that with $R_{12}=0.6 R_{11}$. Fluid A exhibits microphase separation, and is similar to the fluid described in Ref. [12]. This mixture exhibits 1-2 ordering, i.e. where a particle of one species preferentially has particles of the opposite species as nearest neighbours [12. The second mixture, which we denote "fluid B", is that with $R_{12}=1.1 R_{11}$. Fluid B exhibits macrophase or bulk phase separation. This is driven by the fact that $R_{12}>R_{11}=R_{22}$ [1]. The bulk critical point for this mixture is at a total density $\rho R_{11}^{3}=0.54$ and, by symmetry, at concentration $x_{1}=x_{2}=0.5$.

When the GCM fluid density becomes sufficiently high, the following mean-field Helmholtz free energy functional is rather accurate [9, 10, 11]:

$F\left[\left\{\rho_{i}(\mathbf{r})\right\}\right]=F_{i d}\left[\left\{\rho_{i}(\mathbf{r})\right\}\right]+\frac{1}{2} \sum_{i, j} \int \mathrm{d} \mathbf{r} \int \mathrm{d} \mathbf{r}^{\prime} \rho_{i}(\mathbf{r}) \rho_{j}\left(\mathbf{r}^{\prime}\right) u_{i j}\left(\left|\mathbf{r}-\mathbf{r}^{\prime}\right|\right)+\sum_{i} \int \mathrm{d} \mathbf{r} V_{i}(\mathbf{r}) \rho_{i}(\mathbf{r}),(1)$ 

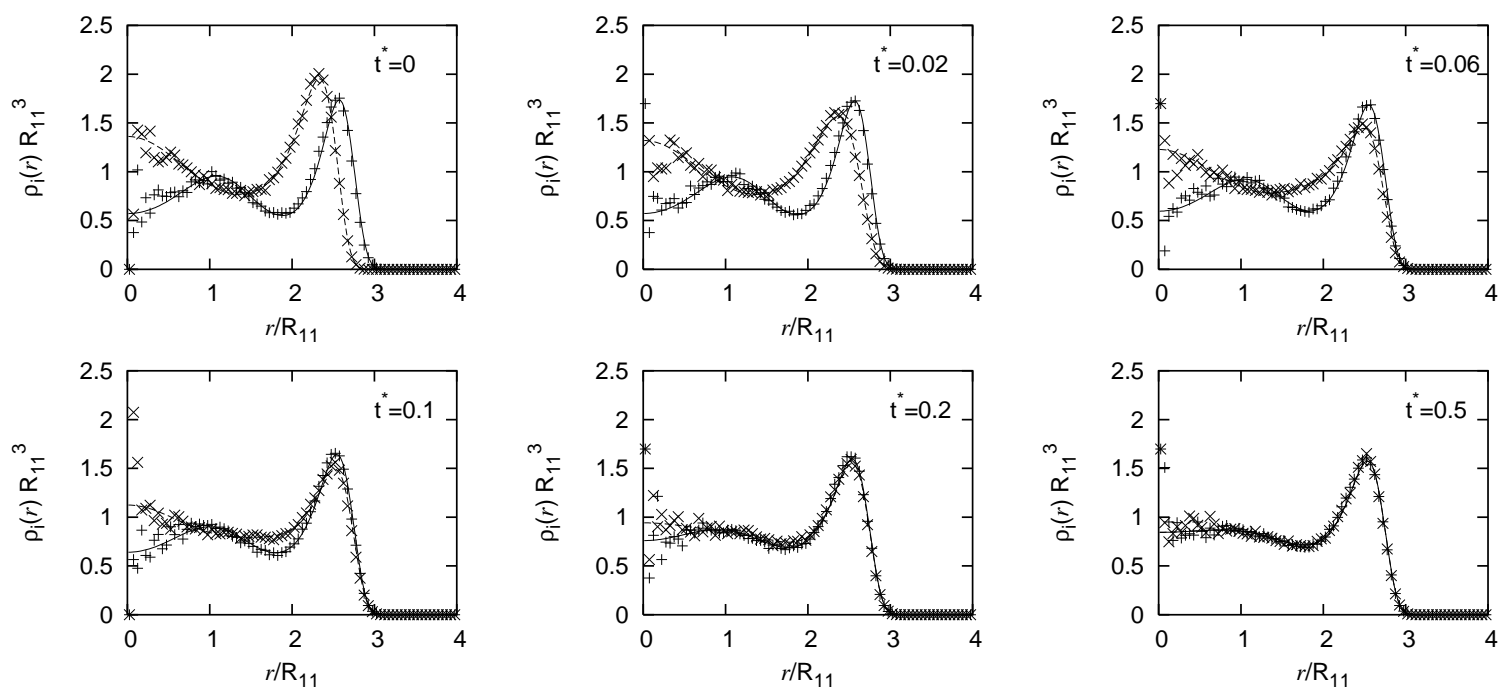

Figure 1. Fluid A density profiles $\rho_{i}(r, t)$ (solid line: DDFT results for species 1, dashed line: species 2; symbols are BD results, $(+)$ for species $1,(\times)$ for species 2$)$ for $N_{1}=N_{2}=100$ particles, which is initially $(t<0)$ at equilibrium confined in (asymmetric) external potentials with $E_{1}=10 k_{B} T, E_{2}=20 k_{B} T$ and $\mathcal{R}=3 R_{11}$. At $t=0, E_{2}$ is suddenly reduced to $E_{2}=10 k_{B} T$, giving symmetrical confinement. The profiles are plotted for various $t^{*}=k_{B} T \Gamma R_{11}^{2} t$. This model fluid exhibits microphaseseparation: the initial configuration has an 'onion' structure, but on enforcing the symmetry in the external potentials, any signature of this structure in the one body density profiles disappears as time progresses.

where $\left\{\rho_{i}(\mathbf{r})\right\}$ are the fluid one body density profiles, $V_{i}(\mathbf{r})$ is the external potential for particles of species $i$ and $F_{i d}$ is the ideal gas contribution to the free energy [1].

For a fluid of $N=N_{1}+N_{2}$ Brownian (colloidal) particles, one can approximate the equations of motion using the following stochastic Langevin equations of motion: $\Gamma_{i}^{-1} \mathrm{~d} \mathbf{r}_{n}(t) / \mathrm{d} t=-\nabla_{n} U\left(\mathbf{r}^{N}, t\right)+\mathbf{G}_{n}(t)$, where $\mathbf{r}_{n}$ is the position of the $n^{t h}$ particle, $\Gamma_{i}^{-1}$ is a friction constant for particles of species $i$ (we assume $\Gamma_{1}=\Gamma_{2}=\Gamma$ ), $U\left(\mathbf{r}^{N}, t\right)$ is the potential energy and $\mathbf{G}_{n}(t)$ is a stochastic white noise term - see Refs. [2, 4, 7] for more details. From such equations of motion, averaging over all realisations of the stochastic noise, one can obtain the following equations for the time evolution of the ensemble average one body density profile [2, 3, 4, 7]:

$$
\frac{\partial \rho_{i}(\mathbf{r}, t)}{\partial t}=\Gamma_{i} \nabla \cdot\left[\rho_{i}(\mathbf{r}, t) \nabla\left(\frac{\delta F\left[\left\{\rho_{i}(\mathbf{r}, t)\right\}\right]}{\delta \rho_{i}(\mathbf{r}, t)}\right)\right],
$$

where the Helmholtz free energy functional $F\left[\left\{\rho_{i}(\mathbf{r}, t)\right\}\right]$ is given by the equilibrium functional with the equilibrium density profiles $\left\{\rho_{i}(\mathbf{r})\right\}$ replaced by the set of nonequilibrium profiles $\left\{\rho_{i}(\mathbf{r}, t)\right\}$. We solve the DDFT (2) for the binary GCM confined in spherically symmetric external potentials of the form [5, 7]: $V_{i}(r)=E_{i}(r / \mathcal{R})^{10}$, where $r$ is the distance from the origin, $E_{i}$ is an energy scale and the length-scale $\mathcal{R}$ (cavity radius) is the same for both species of particles.

In Fig. 1 we display the results for $N_{1}=N_{2}=100$ particles of each species of a 

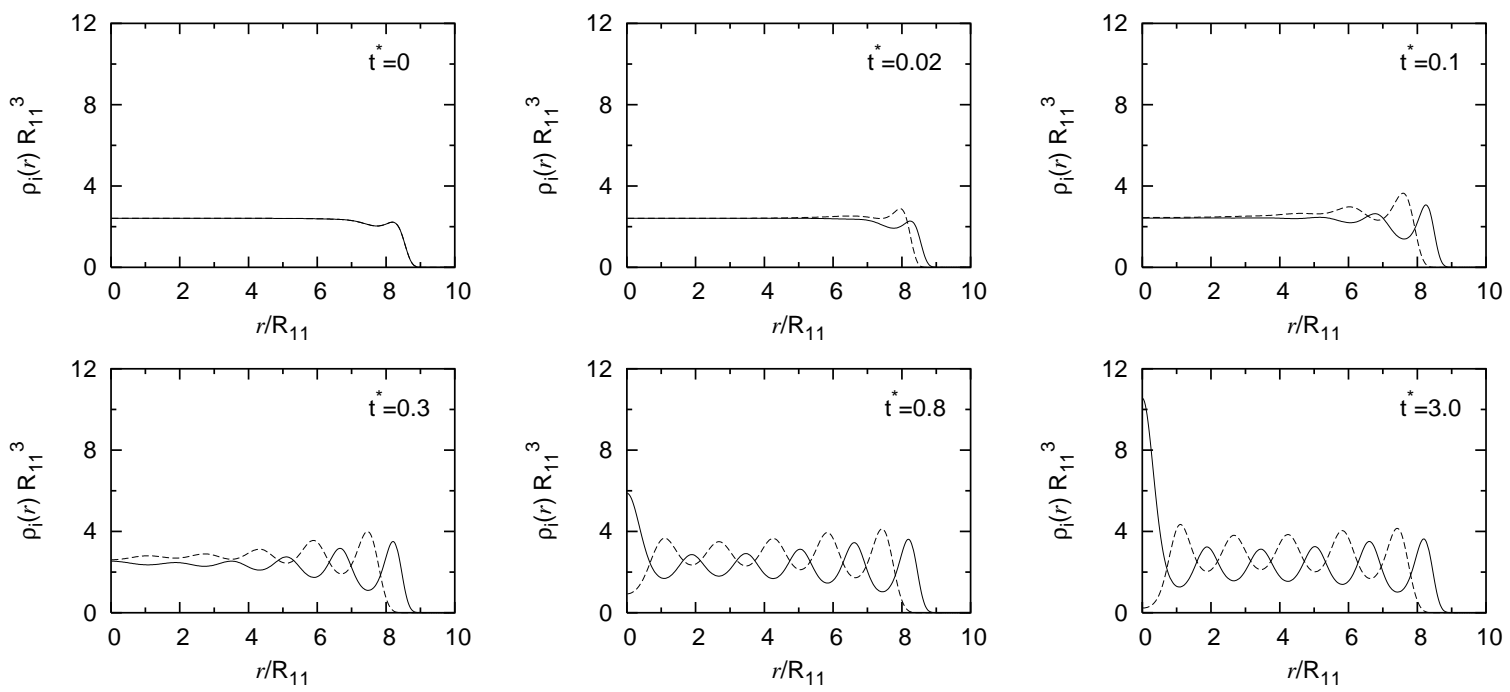

Figure 2. Fluid A density profiles $\rho_{i}(r, t)$ (solid line: species 1, dashed line: species 2) for $N_{1}=N_{2}=6000$ particles. Initially $(t<0)$ the fluid is at equilibrium confined in symmetric external potentials with $E_{2}=E_{1}=10 k_{B} T$ and $\mathcal{R}=8 R_{11}$. At $t=0, E_{2}$ is suddenly increased to $E_{2}=20 k_{B} T$, breaking the symmetry. The profiles are plotted for various $t^{*}=k_{B} T \Gamma R_{11}^{2} t$. Initially there was no signature of microphase separation in the density profiles, but after breaking the symmetry, the fluid profiles develop an 'onion' structure.

mixture of fluid A. Initially $(t<0)$ the external potential parameters $E_{2} \neq E_{1}$ and the fluid one body density profiles exhibit 1-2 ordering (microphase separation) in the cavity. Then at $t=0$ the external potentials are made symmetric: $E_{1}=E_{2}$. At equilibrium, for such symmetric external potentials, the one body density profiles for each species must be the same since $N_{1}=N_{2}$. We see in Fig. 1 that the density profiles quickly evolve to give the same final profiles for each species. We also display the results from $\mathrm{BD}$ simulations, which show extremely good agreement with the results from the DDFT, giving confidence in the DDFT approximation. In Fig. 2 we display DDFT results for a much larger number of particles. The external potentials for $t<0$ are symmetric and therefore the ensemble average density profiles at $t=0$ are the same for both species. At $t=0$ the symmetry in the external potentials is broken, such that particles of species 1 are favoured by the walls of the cavity. The one body density profiles then evolve towards equilibrium density profiles that display the microphase ordering in the fluid 12. Note that this does not mean that there was no microphase separation for $t<0$ (the average total density and composition has not changed); if one were to look at a 'snapshot' of the particle configurations at a time $t<0$ one would indeed see microphase ordering. We illustrate this below for fluid B.

In Fig. [3 we display the results for $N_{1}=N_{2}=100$ particles for fluid B. Initially $(t<0)$ the external potential parameters $E_{2}=E_{1}$ and the fluid one body density profiles are the same for each species. At $t=0$ the symmetry in the external potentials is broken: $E_{1} \neq E_{2}$, such that the cavity walls now favour species 1 . The equilibrium 

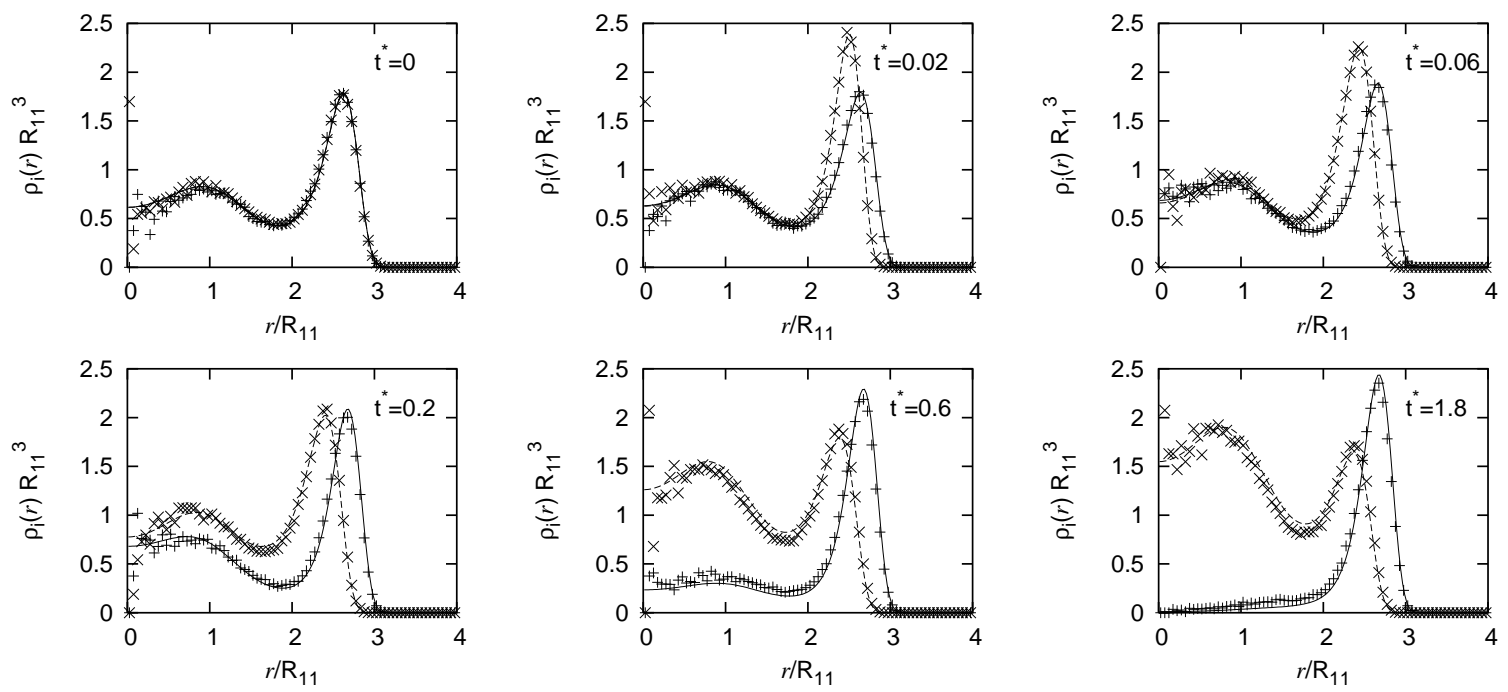

Figure 3. As in Fig. 团 but for fluid B with $N_{1}=N_{2}=100$. The initial density profiles are those for symmetric external potentials: $E_{1}=E_{2}=10 k_{B} T$. At $t=0$, $E_{2} \rightarrow 20 k_{B} T$. The subsequent profiles then show the signature of phase separation.

density profiles for the asymmetric external potentials then show that the fluid does indeed exhibit the signature of bulk phase separation. We also display the results from BD simulations, which show extremely good agreement with the results from the DDFT. In Fig. 4 we display the DDFT results for fluid B with a larger number of particles. Once again the external potentials for $t<0$ are symmetric and therefore the ensemble average density profiles at $t=0$ are the same for both species. At $t=0$ the symmetry is broken and the one body density profiles then evolve towards ones that show the fluid-fluid phase separation. Interestingly, this evolution to the final equilibrium density profiles proceeds via an intermediate state where the fluid is rich in species 1 at both the centre and close to the wall of the cavity. Then, as time proceeds, species 1 particles at the centre of the cavity diffuse to the outside, since the intermediate state has two fluid-fluid interfaces which has a higher free energy cost than the final equilibrium configuration with only one fluid-fluid interface. Despite the fact that the $t=0$ profiles show no sign of phase separation, in the final frame of Fig. 4 we see that in a particular configuration of the particles that there is phase separation in the cavity - i.e. the $t=0$ profiles in Fig. 4 are the result of ensemble averaging over strongly asymmetric configurations. This implies that in this larger cavity there are strong fluctuation effects over the whole cavity, which are neglected in the present mean field DFT treatment [14. We therefore expect the DDFT results of Fig. 4 to be less reliable than those of Fig. 3.

We conclude by emphasising that although the initial $(t=0)$ one body density profiles in Figs. 2- 4 show no obvious sign of phase separation, this does not mean that there will be no signature of phase separation in a particular equilibrium configuration of the particles subject to symmetric external potentials. However, in the ensemble average, the one body density profiles must be the same and therefore show no signature 

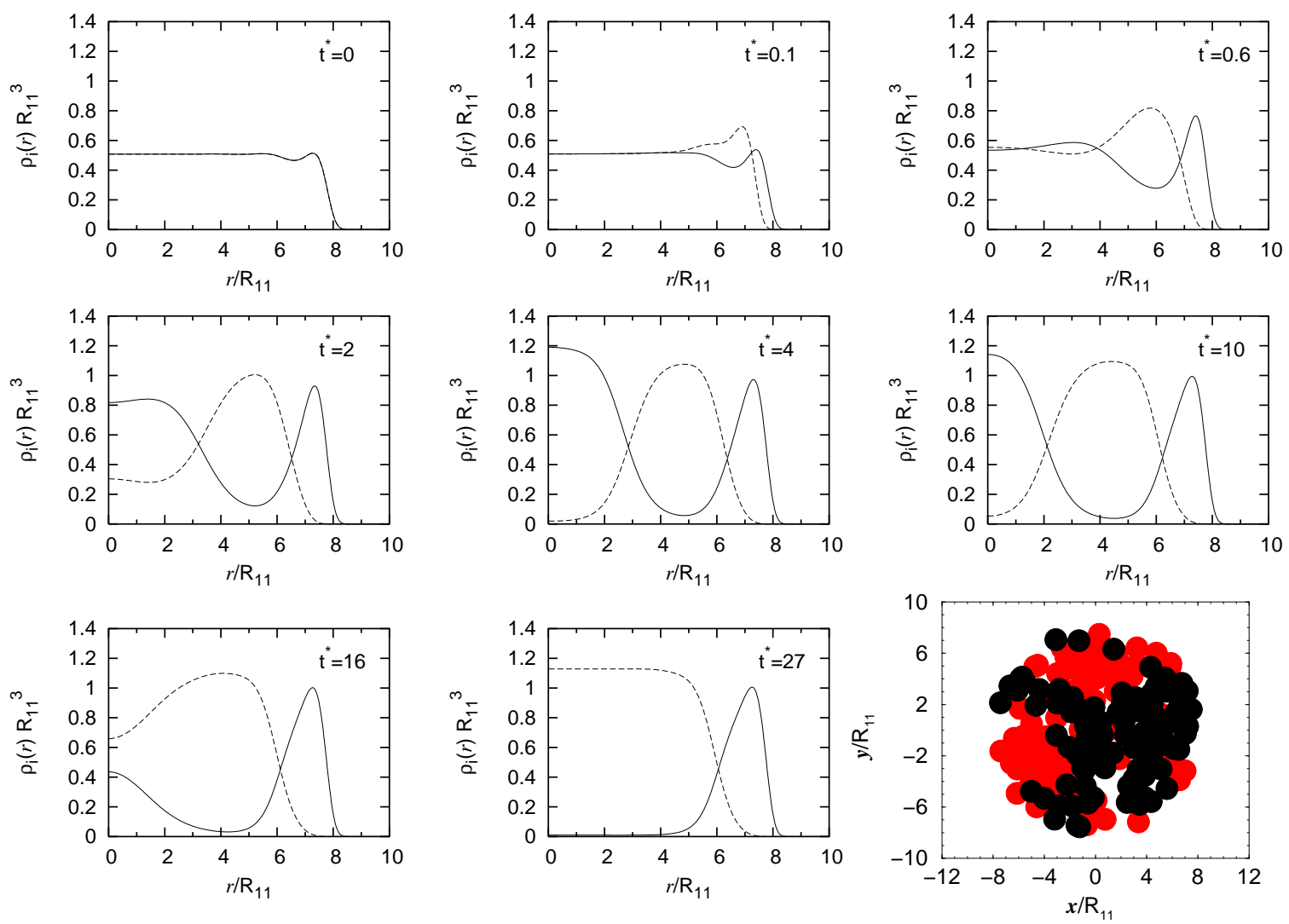

Figure 4. As in Fig. 22 but now for fluid B with $N_{1}=N_{2}=1000$ particles. Initially there is no signature of phase separation in the density profiles, but after breaking the symmetry in the external potentials, there is. Note the intermediate profiles exhibit two fluid-fluid interfaces (rather than one). In the final frame we display a slice (particles with $-0.5 R_{11}<z<0.5 R_{11}$ ) from an equilibrium configuration $t<0$. Even though the one body density profiles $(t=0)$ show no signature of phase separation, this configuration does.

of phase separation. Such effects demonstrate an important aspect of the DDFT: this is a theory for the ensemble average fluid one body density profiles [13] and therefore, as with equilibrium DFT, the fluid density profiles must exhibit the symmetry of the confining external potentials.

Acknowledgements: I thank Bob Evans for a critical reading of the manuscript and I gratefully acknowledge the support of EPSRC under grant number GR/S28631/01.

\section{References}

[1] Evans R 1992, in Fundamentals of Inhomogeneous Fluids, ed. Henderson D, Dekker, New York

[2] Marini Bettolo Marconi U and Tarazona P 1999 J. Chem. Phys. 1108032

[3] Marini Bettolo Marconi U and Tarazona P 2000 J. Phys.: Condens. Matter 12 A413

[4] Archer A J and Evans R 2004 J. Chem. Phys. 1214246

[5] Dzubiella J and Likos C N 2003 J. Phys.: Condens. Matter 15 L147

[6] Penna F, Dzubiella J and Tarazona P 2003 Phys. Rev. E 68061407

[7] Archer A J 2005 J. Phys.: Condens. Matter 171405 
DDFT: phase separation in a cavity and the influence of symmetry

[8] Rex M, Löwen H, and Likos C N 2005 Phys. Rev. E 72021404

[9] Likos C N 2001 Phys. Rep. 348267

[10] Louis A A, Bolhuis P G and Hansen J-P 2000 Phys. Rev. E 627961

[11] Archer A J and Evans R 2001 Phys. Rev. E 64041501

[12] Archer A J, Likos C N and Evans R 2004 J.Phys.: Cond. Matter 16 L297

[13] Archer A J and Rauscher M 2004 J.Phys. A: Math. Gen. 379325

[14] Reguera D and Reiss H 2004 J. Chem. Phys. 1202558 